\title{
Correlation between functional outcome and the SAMEO-ATO framework
}

\author{
Vito Pontillo ${ }^{1,3}$ (1) $\cdot$ Marialessia Damiani $^{1} \cdot$ Giusi Graziano $^{2} \cdot$ Nicola Quaranta $^{1}$ (i)
}

Received: 28 April 2021 / Accepted: 13 July 2021 / Published online: 26 July 2021

(c) The Author(s) 2021

\begin{abstract}
Purpose To evaluate the recently proposed SAMEO-ATO framework for middle ear and mastoid surgery, by correlating it with the functional outcome in a large cohort of patients operated for middle ear and mastoid cholesteatoma in a tertiary referral center.

Methods We retrospectively included all surgeries for middle ear and mastoid cholesteatoma undergone in our Department between January 2009 and December 2014, by excluding revision surgeries, congenital and petrous bone cholesteatoma. All surgeries were classified according to the SAMEO-ATO framework. The post-operative air bone gap (ABG) was calculated and chosen as benchmark parameter for the correlation analysis.

Results 282 consecutive surgeries for middle ear and mastoid cholesteatoma were released in the study period on a total of 273 patients, with a mean age of 41.2 years. All patients were followed for an average period of 55.3 months. $54 \%$ of patients underwent M2c mastoidectomy (Canal Wall Down, CWD), while the remaining underwent Canal Wall Up (CWU) procedures, being M1b2a mastoidectomy the most common one (33\%). Mean pre-operative and post-operative ABGs were 29.2 and $23.5 \mathrm{~dB}$, with a significant improvement $(p<0.0001)$. 'Mastoidectomy' and 'Ossicular reconstruction' parameters of SAMEO-ATO showed significant association with postoperative ABG, with smaller residual gaps for the classes $\mathrm{Mx}$ and On, and worse hearing results for M3a and Ox.

Conclusion Our results show the utility of SAMEO-ATO framework, and in particular of ' $M$ ' (Mastoidectomy) and 'O' (Ossicular reconstruction) parameters, in predicting the hearing outcome.
\end{abstract}

Keywords SAMEO-ATO $\cdot$ Hearing $\cdot$ Cholesteatoma $\cdot$ Surgery $\cdot$ Tympanoplasty

\section{Introduction}

Cholesteatoma is a mass of keratinizing squamous epithelium located in the middle ear and mastoid that requires surgical treatment. To facilitate the comparison of surgical outcomes and identify prognostic factors, the European Academy of Neurotology and the Japan Otological Society have recently published a joint consensus statement on

Vito Pontillo

pontillovito@gmail.com

1 Otolaryngology Unit, Department of BMS, Neuroscience and Sensory Organs, University of Bari, Bari, Italy

2 Biomedical Sciences and Human Oncology Department, University of Bari, Bari, Italy

3 UOC Otorinolaringoiatria Universitaria, Azienda Ospedaliero-Universitaria Consorziale Policlinico Di Bari, Piazza Giulio Cesare 11, 70124 Bari, Italy the definition, classification and staging of middle ear and mastoid cholesteatoma [1]. Other classification systems have been since proposed, such as the "STAMCO" [2] and the "ChOLE" [3]. All the proposed systems describe in detail the characteristics of the cholesteatoma, but none of them takes into account the surgical technique used for its removal.

Surgical treatment of middle ear and mastoid cholesteatoma has been traditionally classified into Canal Wall Up (CWU) and Canal Wall Down (CWD) tympanoplasty [4, 5]; however, the advent of some technical refinements, such as mastoid obliteration $[6,7]$ and endoscopic ear surgery (EES) [8], has increased the number of surgical techniques and the variables that can affect the surgical outcome.

In 2018, the International Otology Outcome Group (IOOG) proposed a framework that aimed at the categorization of tympano-mastoid surgery [9], with the purpose of favoring the pooling of surgical data in a single large 
database. The SAMEO-ATO framework categorizes both mastoid and middle ear surgery. The acronym SAMEO stands for Stage of Surgery, Approach, Mastoidectomy, External ear canal reconstruction and Obliteration of the mastoid cavity; the acronym ATO refers to Access to middle ear, Tympanic membrane and Ossicular chain surgical management. In the www.ioog.net website the complete framework is available with clear diagrams and explanation. Recently, ten Tije et al. [10] assessed the value of the SAMEO-ATO classification in the description of the surgery in a multi-centric Dutch cohort and concluded that "the newly proposed classification seems to be more detailed in the registration of surgical procedures that surgeons are currently used to". One of the theoretical advantages of such classification is that it may allow a more precise comparison of surgical outcomes and may help to identify factors that affect the outcome.

The aim of the present study was to assess the value of the SAMEO-ATO classification by retrospectively correlating its stages with the hearing outcome in a group of patients surgically treated for middle ear cholesteatoma in a tertiary referral center.

\section{Materials and methods}

The study group includes 273 patients affected by middle ear and mastoid cholesteatoma and operated on in our Department between January 2009 and December 2014. All patients that had undergone previous surgery or affected by congenital or petrous bone cholesteatoma were excluded. Nine subjects underwent bilateral intervention; therefore, the total number of surgeries was 282 .

Mean age at surgery was 41.2 years (range $3-82$ years), $152(55.6 \%)$ were males and $121(44.3 \%)$ females. 146 surgeries were performed on the left side and 136 on the right side. Average post-operative follow-up was $55.3 \pm 33.5$ months (range 6-136 months).

Demographic data, intra-operative findings, surgical technique, post-operative anatomical and functional findings were all recorded in an electronic database.

The intra-operative extension of cholesteatoma and the presence of complications were evaluated according to two staging systems, EAONO/JOS [1] and STAMCO [2]. Furthermore, the analysis of the operative notes allowed the retrospective classification of the surgical technique according to the SAMEO-ATO framework [9]. In the analysis of hearing results, M3a mastoidectomy was excluded, while $\mathrm{M} 2 \mathrm{~b}$ and M2c mastoidectomies were considered as CWD procedures and all the remaining cases were considered as CWU tympanoplasties. The guidelines of the Committee on Hearing and Equilibrium of the American Academy of Otolaryngology Head and Neck Surgery [11] were followed and the pure-tone average (PTA) was calculated as the mean of $0.5,1,2$ and $4 \mathrm{kHz}$ thresholds. Air-Bone Gaps (ABG) were calculated from air conduction (AC) and bone conduction (BC) thresholds. In the evaluation of functional outcome, the postoperative $\mathrm{ABG}$ was chosen as benchmark parameter and the surgical program was considered 'incomplete' in patients who refused the planned second-stage surgery. All patients signed an informed consent, and the work was performed in accordance with the principles of the 1983 Declaration of Helsinki. Approval was obtained by the local EC.

\section{Statistical analysis}

Categorical or dichotomous variables were expressed as absolute number and percentage $(N, \%)$. Continuous variables were expressed as mean \pm standard deviation $(\mu \pm S D)$. Chi-square or Fisher's exact tests and Mann-Whitney test were used to compare categorical and continuous variables, respectively. Wilcoxon test was used to assess the postoperative changes in terms of ABG. A $p$ value $<0.05$ was considered statistically significant. The software $\mathrm{R}$ (version 3.5.2) was used for statistical analysis.

\section{Results}

Table 1 reports the classification of the cholesteatomas according to the EAONO-JOS and STAMCO classifications; in all tables, data for the whole group as well as for the CWU and CWD tympanoplasty groups are reported. According to the EAONO/JOS classification, most of the patients were in stage 2 (cholesteatoma involving two or

Table 1 Distribution of stages according to the EAONO-JOS and STAMCO cholesteatoma classifications in the total population and in CWU or CWD groups

\begin{tabular}{lllll}
\hline & Stage & $\begin{array}{l}\text { Tot. population } \\
N(\%)\end{array}$ & $\begin{array}{l}\text { CWU } \\
N(\%)\end{array}$ & $\begin{array}{l}\text { CWD } \\
N(\%)\end{array}$ \\
\hline EAONO/JOS & $\mathbf{1}$ & $51(18.1)$ & $35(27.3)$ & $16(10.4)$ \\
& $\mathbf{2}$ & $197(69.9)$ & $88(68.8)$ & $109(70.8)$ \\
& $\mathbf{3}$ & $32(11.3)$ & $5(3.9)$ & $27(17.5)$ \\
STAM & $\mathbf{4}$ & $2(0.7)$ & $0(0)$ & $2(1.3)$ \\
& $\mathbf{1}$ & $50(17.7)$ & $33(25.8)$ & $17(11)$ \\
& $\mathbf{2}$ & $56(19.9)$ & $23(18)$ & $33(21.5)$ \\
$\mathbf{C}$ & $\mathbf{3}$ & $176(62.4)$ & $72(56.2)$ & $104(67.5)$ \\
& $\mathbf{n}$ & $248(87.9)$ & $123(96.1)$ & $125(81.2)$ \\
& $\mathbf{1}$ & $32(11.3)$ & $5(3.9)$ & $27(17.5)$ \\
$\mathbf{O}$ & $\mathbf{2}$ & $2(0.8)$ & $5(3.9)$ & $27(17.5)$ \\
& $\mathbf{n}$ & $51(18.01)$ & $26(20.3)$ & $25(16.2)$ \\
& $\mathbf{1}$ & $111(39.4)$ & $53(41.4)$ & $58(37.7)$ \\
& $\mathbf{2}$ & $81(28.7)$ & $41(32)$ & $40(26)$ \\
& $\mathbf{3}$ & $39(13.8)$ & $8(6.3)$ & $3(20.1)$ \\
\hline
\end{tabular}

Bold values: statistically significant results (p-value<0.005) 
more sites). Statistical analysis showed that patients in stage 3 (cholesteatoma with extracranial complications) and 4 (cholesteatoma with intracranial complications) were preferentially treated by CWD procedures $(p<0.001)$.

According to the STAMCO classification, two-thirds of patients were classed as STAM 3 (cholesteatoma in three locations or in one difficult site). Statistical analysis showed that CWD procedures were significantly more frequent in more extensive cholesteatomas (higher STAM stages) $(p=0.0055)$, in case of complications $(p=0.002)$ and greater ossicular chain involvement $(p=0.0092)$.

In Table 2, the classification of the surgeries according to the SAMEO/ATO framework is reported. Almost 70\% of interventions were staged, and the approach was retroauricular in $90 \%$ of cases. $54 \%$ of patients underwent an M2c mastoidectomy, while the remaining $45 \%$ underwent CWU procedures, being a M1b2a mastoidectomy the most common one $(33 \%)$. The ear canal was never reconstructed entirely, while in case of M2a mastoidectomy, the scutum was usually reconstructed with cartilage or bone paste. In M2c, the mastoid was partially obliterated with bone paste in most of the cases (131/152).

Table 2 reports the classification of surgeries according to the ATO framework. For the reconstruction of the ossicular chain, homologous costal cartilage was used in 51.6\% of cases, autologous ossicles in $25.8 \%$, tragal cartilage in $15.1 \%$, titanium prosthesis in $6.9 \%$ and bone paste in $0.6 \%$.
In Table 3, hearing results in terms of AC PTA and ABG are reported for the pre- and post-operative periods for the whole population and for the CWU and CWD groups. In the entire sample, a significant improvement in the post-operative period was found both for AC-PTA and ABG, while average $\mathrm{BC}$ did not change significantly. In the post-operative period, almost $50 \%$ of patients showed an $A B G \leq 20 \mathrm{~dB}$.

In the CWU group, both AC-PTA and ABG improved after surgery, while in the CWD group, only ABG improved. Moreover, significant better post-operative AC-PTA and ABG were found in the CWU group compared to CWD. $54.8 \%$ and $38.4 \%$ of patients presented a post-operative $\mathrm{ABG} \leq 20 \mathrm{~dB}$ in the CWU and CWD groups, respectively, being the significant difference $(p=0.019)$.

As reported in Table 3, long-term pure-tone audiometry, recorded at an average time of $4.3 \pm 2.6$ years from surgery (min 2, max 10 years), showed a deterioration of AC-PTA $(p<0.0001)$ and $\mathrm{ABG}(p=0.001)$ in the CWU group, while results were more stable in the CWD group.

Residual cholesteatoma was found to be significantly more frequent in the CWU group (17.2 vs $3.9 \%, p=0.0002$ of CWD), as well as recurrent cholesteatoma (7 vs $0 \%$ of CWD, $p=0.001)$. Other long-term anatomical complications, such as TM retraction, granulations or epidermoid cysts, were found in $8.6 \%$ of patients submitted to a CWU and in $7.1 \%$ of those who had undergone a CWD procedure, with no significant difference between the two groups
Table 2 Surgery classification according to the SAMEO/ATO framework

\begin{tabular}{|c|c|c|c|c|c|}
\hline Category & Class & $N(\%)$ & Category & Class & $N(\%)$ \\
\hline \multirow{2}{*}{$\begin{array}{l}\mathbf{S} \\
\text { (Stage of surgery) }\end{array}$} & S1 & $90(31.9)$ & \multirow{4}{*}{$\begin{array}{l}\text { A } \\
\text { (Access to the middle ear) }\end{array}$} & $A x$ & $267(94.7)$ \\
\hline & S2 & $192(68.1)$ & & A1 & $5(1.8)$ \\
\hline \multirow{4}{*}{$\begin{array}{l}\text { A } \\
\text { (Approach) }\end{array}$} & A1 & 0 & & A2 & $10(3.5)$ \\
\hline & $\mathbf{A 2}$ & $29(10.3)$ & & A3 & 0 \\
\hline & $\mathbf{A 3}$ & 0 & \multirow{5}{*}{$\begin{array}{l}\mathbf{T} \\
\text { (Tympanic membrane) }\end{array}$} & $\mathbf{T x}$ & $2(0.7)$ \\
\hline & A4 & 253 (89.7) & & Tn & 0 \\
\hline \multirow{10}{*}{$\begin{array}{l}\mathbf{M} \\
\text { (Mastoidectomy) }\end{array}$} & $\mathbf{M x}$ & $14(5.0)$ & & T1 & $4(1.4)$ \\
\hline & M1a & 0 & & $\mathbf{T 2}$ & $32(11.3)$ \\
\hline & M1b & 0 & & T3 & 244 (86.6) \\
\hline & M2a & $15(5.3)$ & \multirow{13}{*}{$\begin{array}{l}\text { O } \\
\text { (Ossicular chain) }\end{array}$} & Ox & $69(24.5)$ \\
\hline & M2b & 0 & & On & $23(8.2)$ \\
\hline & M2c & $152(53.9)$ & & Osi & $5(1.8)$ \\
\hline & $M 1 a+2 a$ & $6(2.1)$ & & Osm & 0 \\
\hline & $M 1 b+2 a$ & $93(33.0)$ & & Ost & $87(30.9)$ \\
\hline & M3a & $2(0.7)$ & & Osd & $26(9.2)$ \\
\hline & M3b & 0 & & Ofi & 0 \\
\hline \multirow{3}{*}{$\begin{array}{l}\mathbf{E} \\
\text { (Ear canal reconstruction) }\end{array}$} & $\mathbf{E x}$ & $168(59.6)$ & & Ofm & 0 \\
\hline & E1 & 0 & & Oft & $68(24.1)$ \\
\hline & E2 & $114(40.4)$ & & Ofd & $4(1.4)$ \\
\hline \multirow{3}{*}{$\begin{array}{l}\mathbf{O} \\
\text { (Obliteration) }\end{array}$} & $\mathbf{O x}$ & $150(53.2)$ & & Ovi & 0 \\
\hline & 01 & $101(35.8)$ & & Ovm & 0 \\
\hline & O2 & $31(11.0)$ & & Ovt & 0 \\
\hline
\end{tabular}

Bold values: statistically significant results ( $\mathrm{p}$-value $<0.005$ ) 
Table 3 Functional results in the whole population and in the CWU or CWD groups

\begin{tabular}{lllll}
\hline & Total & CWU & CWD & $\begin{array}{l}p \text { value } \\
\text { (CWU vs CWD) }\end{array}$ \\
\hline Delta-BC PTA $(\mu \pm \mathrm{DS})$ & $-2.4 \pm 11.5$ & $-0.8 \pm 7.1$ & $-3.8 \pm 14.2$ & 0.1303 \\
Pre-op PTA $_{\mathrm{AC}}(\mu \pm \mathrm{DS})$ & $52.3 \pm 20.2$ & $50.2 \pm 18.9$ & $54.1 \pm 21.2$ & 0.2229 \\
Post-op PTA $_{\mathrm{AC}}(\mu \pm \mathrm{DS})$ & $48.6 \pm 23.0$ & $42.6 \pm 19.5$ & $54.4 \pm 24.7$ & $\mathbf{0 . 0 0 0 5}$ \\
Last PTA $_{\mathrm{AC}}(\mu \pm \mathrm{DS})$ & $50.2 \pm 21.9$ & $47.8 \pm 20.5$ & $52.8 \pm 23.1$ & 0.236 \\
$\boldsymbol{p}$ value $($ pre- vs post-op) & $\mathbf{0 . 0 0 1 2}$ & $<\mathbf{0 . 0 0 0 1}$ & 0.4687 & \\
$\boldsymbol{p}$ value $($ post-op vs last) & $<\mathbf{0 . 0 0 0 1}$ & $<\mathbf{0 . 0 0 0 1}$ & 0.054 & \\
Pre-op ABG $(\mu \pm \mathrm{DS})$ & $29.2 \pm 12.1$ & $29.0 \pm 12.4$ & $29.4 \pm 12.0$ & 0.6265 \\
Post-op ABG $(\mu \pm \mathrm{DS})$ & $23.5 \pm 13.7$ & $20.8 \pm 12.0$ & $26.2 \pm 14.7$ & $\mathbf{0 . 0 0 5 4}$ \\
Last ABG $(\mu \pm \mathrm{DS})$ & $25.3 \pm 13.7$ & $25.8 \pm 14.2$ & $24.7 \pm 13.2$ & 0.704 \\
$\boldsymbol{p}$ value $($ pre- vs post-op) & $<\mathbf{0 . 0 0 0 1}$ & $<\mathbf{0 . 0 0 0 1}$ & $\mathbf{0 . 0 0 5 3}$ & \\
$\boldsymbol{p}$ value $($ post-op vs last) & $\mathbf{0 . 0 0 2}$ & $\mathbf{0 . 0 0 1}$ & 0.49 & \\
Post-op air bone gap & & & & \\
$\mathrm{ABG} \leq 20 \mathrm{~dB} n(\%)$ & $98(46.7)$ & $57(54.8)$ & $41(38.7)$ & $\mathbf{0 . 0 1 9}$ \\
$\mathrm{ABG}>20 \mathrm{~dB} n(\%)$ & $112(53.3)$ & $47(45.2)$ & $65(61.3)$ & \\
\hline
\end{tabular}

Bold values: statistically significant results ( $\mathrm{p}$-value $<0.005$ )
( $p=0.652)$. Long-term functional complications, such as dislocation, extrusion or absorption of the ossiculoplasty material, were recorded in 8 cases of CWU (6.25\%) and in 2 cases of CWD (1.3\%), with only a trend towards significance between the two techniques $(p=0.047)$.

Table 4 shows the results of the univariate correlation analysis between post-operative ABG and the pre- and intraoperative factors for the entire population and CWU and CWD groups.

\section{Study group}

None of the cholesteatoma staging systems, except the 'ossicular chain status' at the beginning of surgery of the STAMCO classification, correlated with post-operative hearing outcome; in fact, higher ' $\mathrm{O}$ ' stages were associated with significantly poorer hearing results for the whole population $(p=0.0038)$. Incomplete surgical program negatively correlated with the functional outcome $(p=0.0016)$. In regard to the SAMEO-ATO framework, the Approach, External ear reconstruction, Obliteration, Access to middle ear and Tympanic membrane reconstruction were not evaluated in the statistical analysis, since most of the patients were staged as E2, O1, Ax and T3.

$\mathrm{Mx}$ and M2a classes showed the best hearing results in terms of postoperative $\mathrm{ABG}$, while the worst results concerned the patients submitted to M3c mastoidectomies $(p=0.016)$. CWU tympanoplasties as a group ( $\mathrm{Mx}$, $\mathrm{M} 2 \mathrm{a}, \mathrm{M} 1 \mathrm{a}+2 \mathrm{a}$ and $\mathrm{M} 1 \mathrm{~b}+2 \mathrm{a}$ ) were associated with better post-operative hearing function both in terms of average $\mathrm{ABG}$ and the number of patients with an $\mathrm{ABG}$ better than $20 \mathrm{~dB}$ HL. Comparing the most frequently used mastoidectomy techniques, $\mathrm{M} 1 \mathrm{~b}+2 \mathrm{a}$ showed better post-operative
ABG averages than M2c $(21.7 \pm 12$ vs $25.9 \pm 14.5 \mathrm{~dB}$ HL; $p=0.034)$.

In terms of 'ossicular reconstruction' $(\mathrm{O})$, On (intact chain preservation) and Osi (reconstruction between incus and stapes head in case of limited erosion of the long process of the incus) classes were associated with the lowest ABG (respectively, 13.1 and $19.3 \mathrm{~dB}$ ), while the worst results were registered in cases where no reconstruction was performed $(\mathrm{Ox}$, mean $\mathrm{ABG}=33.7 \mathrm{~dB})$. No significant differences in terms of post-operative ABG were encountered when comparing partial (Osi, Ost, Osd) with total (Oft, Ofd) ossiculoplasty or the materials used for reconstruction. However, long-term post-operative ABG showed significant better values when autologous ossicles had been used for reconstruction $(19.2 \pm 13.8 \mathrm{~dB})$ compared to allogenic costal cartilage $(27.9 \pm 14.2 \mathrm{~dB} ; p=0.008)$ and titanium prosthesis $(32.7 \pm 13.4 \mathrm{~dB} ; p=0.028)$.

A within-groups comparison showed that in CWUT single-stage surgery, intact ossicular chain at the end of surgery (On) and minimal reconstruction (Osi) were all associated with better hearing results. In CWDT, incomplete surgery was a negative prognostic factor, while an intact chain at the end of surgery was associated with better hearing results (Table 4).

\section{Discussion}

In the present study, the SAMEO-ATO classification was correlated with the functional outcome in a large cohort of patients affected by middle ear and mastoid cholesteatoma.

The SAMEO-ATO framework has been recently proposed to categorize the surgical techniques used for the treatment 
Table 4 Univariate correlation analysis between post-operative ABG and pre-operative or intraoperative features

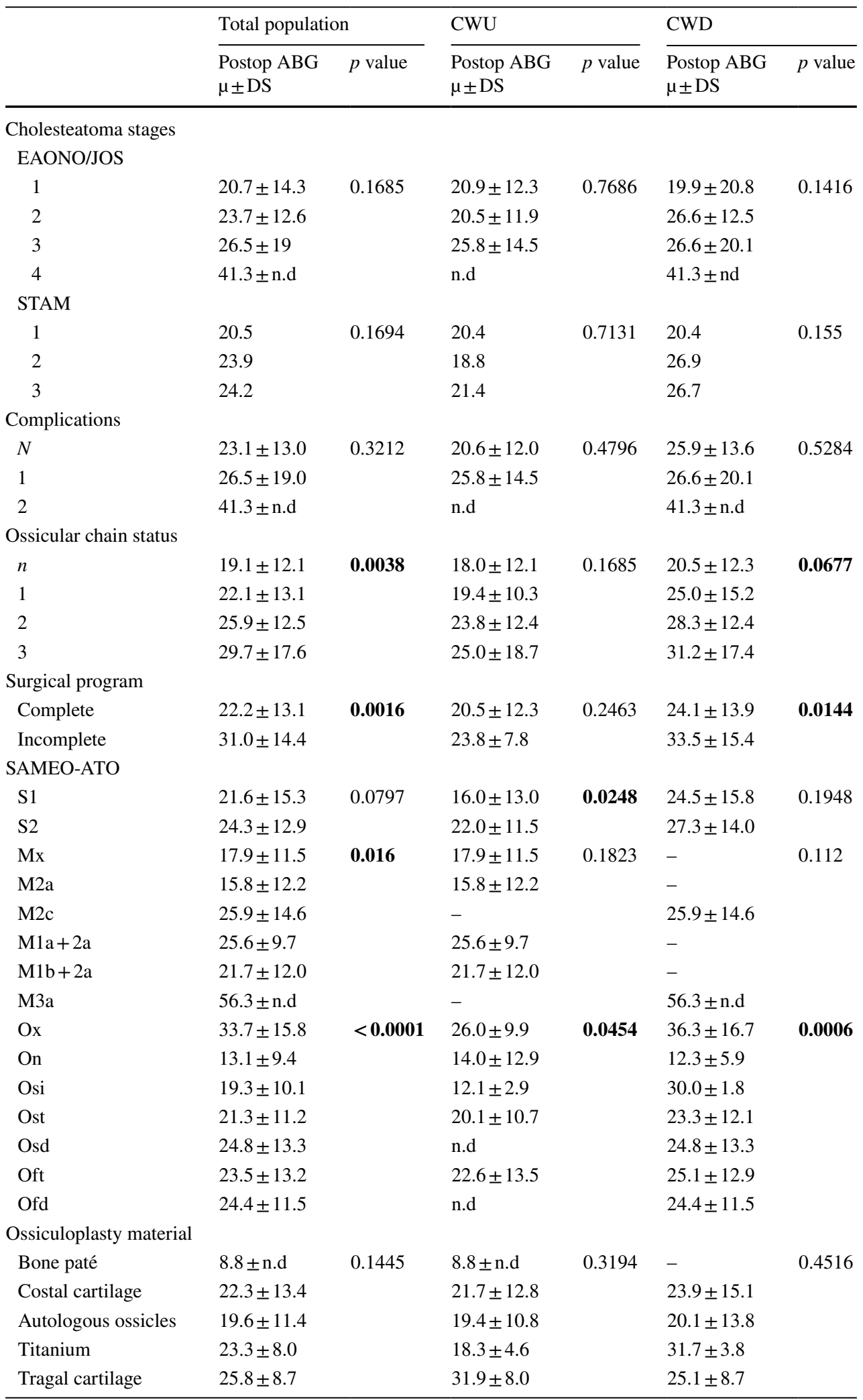

Bold values: statistically significant results ( $\mathrm{p}$-value $<0.005$ ) of cholesteatoma and allow the comparison of surgical results [9]. The role of cholesteatoma staging systems in predicting surgical outcome has been reported by different authors. Van der Tom et al. [12] evaluated the role of the 
EAONO/JOS [1] and STAMCO [2] classifications and demonstrated STAMCO's superiority in predicting both residual and recurrent disease. However, this finding was only referred to patients treated with CWU tympanoplasty, since recurrent and residual cholesteatoma rates were negligible in the CWD group. In terms of hearing results, the authors found, in the CWU group, larger post-operative ABG with increasing stage (both EAONO/JOS and STAM), complications and ossicular chain status; on the contrary, in the CWD group, increasing ossicular chain status correlated with higher AC PTA, with no effect on ABG. Angeli et al. [13] evaluated the prognostic value of the EAONO/JOS classification in terms of recidivism, in a group of patients affected by cholesteatoma and treated with a CWU tympanoplasty. The authors showed that the recidivism rate was higher in children, in case of larger bone canal defect or cholesteatoma located in the supra-tubal recess; however, the prognostic role of the classification was defined as "uncertain" [13]. Ardıç et al. [14] correlated the surgical outcomes with the EAONO/JOS staging system and reported no correlation between the staging system and the recurrence rate. Although they described the number of patients treated with CWU and CWD procedures, the authors did not analyze the role of surgical technique in the recidivism rate. Fukuda et al. [15] reported the short-term hearing results in a small group of patients affected by pars flaccida cholesteatoma and submitted to mastoidectomy and tympanoplasty with cartilage double-block reconstruction on the stapes. The authors classified all the cases with the EAONO/JOS staging system and used a post-operative $\mathrm{ABG}<20 \mathrm{~dB}$ as success criterion, thereby finding that poorer hearing results were associated with higher EAONO/JOS stages and stapes involvement.

It is therefore clear from the existing literature that the pre-operative stage does not consistently predict by itself the post-operative outcome, especially when the surgical technique used to treat cholesteatoma is not taken in consideration.

In the present study, we have correlated the post-operative hearing results both with cholesteatoma staging systems and surgical procedure. In particular, this is the first study that evaluates the correlation of the SAMEO-ATO framework with the hearing outcome. Statistical analysis showed that, in regard to cholesteatoma staging systems, the main factors responsible for the post-operative hearing are a complete surgical program, the status of the ossicular chain at the beginning of surgery and the surgical technique used.

van der Toom et al. [12], using the STAMCO classification, showed that the ossicular chain status at the beginning of surgery was the only factor correlating with hearing outcome.

Our correlation analysis of the SAMEO framework showed that the $\mathrm{M}$ status associated with hearing results; within the ATO framework, the O status showed its value, in particular an intact ossicular chain at the end of the procedure (On) was associated with the best hearing results.

As a group, CWU procedures were associated with better post-operative hearing in terms of AC PTA and ABG compared to CWD procedures. Although these results could be easily explicable in case of no (Mx) or minimal mastoid surgery (M2a) since related to the small size of the cholesteatoma and to the minimal involvement of the ossicular chain, it should be highlighted the fact that even the comparison between M1b2a (mastoidectomy with posterior tympanotomy and scutum reconstruction) and M2c (canal wall down mastoidectomy with partial obliteration) confirmed these findings in our analysis. Similar results were reported by other authors that compared CWU and CWD procedures [16-19]. The presence of a larger middle ear space and a near-normal anatomy allows a more "physiological" reconstruction of the ossicular chain.

As reported in a recent meta-analysis [20], also in the present series, CWUT was associated with significantly higher residual and recurrent cholesteatoma; therefore, the choice between CWUT and CWDT procedures should be balanced also considering the higher risk of recurrence in "closed" procedures.

The preservation of the ossicular chain both in CWU and CWD tympanoplasties was associated with the best hearing results. In case of an intact ossicular chain at the beginning of the procedure, it can be saved using both CWU and CWD techniques. Our group has showed that in case of cholesteatoma located only in the middle ear space, the preservation of the ossicular chain is possible maintaining the posterior wall of the EAC intact, while in case of epitympanic cholesteatoma with extension in the mastoid antrum, the Bondi technique allows the preservation of hearing with minimal recurrence rates [21]. Similar results have been reported by other authors [22-24].

In terms of reconstruction of the ossicular chain, no significant differences were encountered in terms of partial or total reconstruction. Yu et al. [25] performed a meta-analysis of the existing literature and compared the effect of PORPs and TORPs for the ossicular chain reconstruction. PORPs were comprehensively more effective than TORPs in terms of hearing function, but no difference was detected in staged procedures subgroup and in cholesteatoma subgroup, similarly to the present series where most of the surgeries were staged and all the patients were affected by cholesteatoma.

\section{Conclusion}

Cholesteatoma classification and staging systems have gained increased attention in recent years. In particular, EAONO/JOS and STAMCO classifications have demonstrated their utility in various aspects of clinical evaluation, 
but they mostly failed in terms of predictive power both in terms of cholesteatoma recidivism and hearing outcome. This is likely attributable to the fact that none of these classification systems considers the specific surgical features. The SAMEO-ATO framework has been recently proposed to categorize the surgical techniques used for the treatment of cholesteatoma and allows a more direct comparison of surgical results. To our knowledge, this is the first study evaluating the correlation of the SAMEO-ATO framework with the hearing outcome. Our results show the utility of this framework, and in particular of ' $M$ ' (Mastoidectomy) and ' $\mathrm{O}$ ' (Ossicular reconstruction) parameters, in predicting hearing outcome. Besides, the analysis of our series points up the need of an integrated classification system that combines both pathological and surgical evaluations.

Author contributions All the authors have contributed to the diagnostic and therapeutic processes and to the writing of the present paper.

Funding Open access funding provided by Università degli Studi di Bari Aldo Moro within the CRUI-CARE Agreement.

Data availability All data and material are available and can be provided if requested.

Code availability Not applicable.

\section{Declarations}

Conflict of interest The authors have no conflicts of interest to declare.

Ethical approval The authors have no ethical conflicts to disclose. Approval was obtained by the local Ethical Commission.

Consent to participate All patients have given her informed written consent to surgery.

Consent for publication All patients have given her informed written consent for publication of their data.

Open Access This article is licensed under a Creative Commons Attribution 4.0 International License, which permits use, sharing, adaptation, distribution and reproduction in any medium or format, as long as you give appropriate credit to the original author(s) and the source, provide a link to the Creative Commons licence, and indicate if changes were made. The images or other third party material in this article are included in the article's Creative Commons licence, unless indicated otherwise in a credit line to the material. If material is not included in the article's Creative Commons licence and your intended use is not permitted by statutory regulation or exceeds the permitted use, you will need to obtain permission directly from the copyright holder. To view a copy of this licence, visit http://creativecommons.org/licenses/by/4.0/.

\section{References}

1. Yung M, Tono T, Olszewska E, Yamamoto Y, Sudhoff H, Sakagami M, Mulder J, Kojima H, İncesulu A, Trabalzini F, Özgirgin N (2017) EAONO/JOS Joint Consensus Statements on the definitions, classification and staging of middle ear cholesteatoma. J Int Adv Otol 13:1-8. https://doi.org/10.5152/iao.2017.3363

2. Merkus P, Ten Tije FA, Stam M, Tan FML, Pauw RJ (2017) Implementation of the "EAONO/JOS Definitions and classification of middle ear cholesteatoma"'-from STAM to STAMCO. J Int Adv Otol 13:272-275. https://doi.org/10.5152/iao.2017.4049

3. Linder TE, Shah S, Martha AS, Roosli C, Emmett SD (2019) Introducing the "'ChOLE" classification and its comparison to the EAONO/JOS consensus classification for cholesteatoma staging. Otol Neurotol 40:63-72. https://doi.org/10.1097/MAO.00000 00000002039

4. Smyth GD (1985) Cholesteatoma surgery: the influence of the canal wall. Laryngoscope 95(1):92-96. https://doi.org/10.1288/ 00005537-198501000-00022

5. Karmarkar S, Bhatia S, Saleh E, DeDonato G, Taibah A, Russo A, Sanna M (1995) Cholesteatoma surgery: the individualized technique. Ann Otol Rhinol Laryngol 104(8):591-595. https:// doi.org/10.1177/000348949510400801

6. Shea MC Jr, Gardner G Jr, Simpson ME (1972) Mastoid obliteration using homogenous bone chips and autogenous bone paste. Trans Am Acad Ophthalmol Otolaryngol 76(1):160-172

7. Palva T (1973) Operative technique in mastoid obliteration. Acta Otolaryngol 75(4):289-290. https://doi.org/10.3109/00016 487309139718

8. Thomassin JM, Korchia D, Doris JM (1993) Endoscopic-guided otosurgery in the prevention of residual cholesteatomas. Laryngoscope 103(8):939-943. https://doi.org/10.1288/00005537199308000-00021

9. Yung M, James A, Merkus P, Philips J, Black B, Tono T, Linder T, Dornhoffer J, İncesulu A (2018) International otology outcome group and the international consensus on the categorization of tympanomastoid surgery. J Int Adv Otol 14(2):216-226. https://doi.org/10.5152/iao.2018.5553

10. Ten Tije FA, Alkema S, van der Putten L, Koopman JP, Buwalda J, Kramer SE, Pauw RJ, Merkus P, Dutch Cholesteatoma Data Consortium (2021) Evaluation of the SAMEO-ATO surgical classification in a Dutch cohort. Eur Arch Otorhinolaryngol 278(3):653-658. https://doi.org/10.1007/s00405-020-06109-1

11. Committee on Hearing and Equilibrium guidelines for the evaluation of results of treatment of conductive hearing loss. American Academy of Otolaryngology-Head and Neck Surgery Foundation, Inc. (1995). Otolaryngol Head Neck Surg 113(3):186-187. https://doi.org/10.1016/S0194-5998(95) 70103-6.

12. van der Toom H, van der Schroeff MP, Janssen J, Westzaan AM, Pauw RJ (2020) A retrospective analysis and comparison of the STAM and STAMCO classification and EAONO/JOS cholesteatoma staging system in predicting surgical treatment outcomes of middle ear cholesteatoma. Otol Neurotol 41(4):e468-e474. https:// doi.org/10.1097/MAO.0000000000002549

13. Angeli S, Shahal D, Brown CS, Herman B (2020) Predicting recidivism for acquired cholesteatoma: evaluation of a current staging system. Otol Neurotol 41(10):1391-1396. https://doi.org/ 10.1097/MAO.0000000000002823

14. Ardıç FN, Mengi E, Tümkaya F, Kara CO, Bir F (2020) Correlation between surgical outcome and stage of acquired middle ear cholesteatoma: revalidation of the EAONO/JOS staging system. J Int Adv Otol 16(1):34-39. https://doi.org/10.5152/iao.2020.7598

15. Fukuda A, Morita S, Nakamaru Y, Hoshino K, Fujiwara K, Homma A (2019) Short-term hearing prognosis of ossiculoplasty 
in Pars Flaccida cholesteatoma using the EAONO/JOS staging system. J Int Adv Otol 15(1):2-7. https://doi.org/10.5152/iao. 2019.5983

16 Quaranta N, Taliente S, Coppola F, Salonna I (2015) Cartilage ossiculoplasty in cholesteatoma surgery: hearing results and prognostic factors. Acta Otorhinolaryngol Ital 35(5):338-342

17. Dornhoffer JL, Gardner E (2001) Prognostic factors in ossiculoplasty: a statistical staging system. Otol Neurotol 22(3):299-304. https://doi.org/10.1097/00129492-200105000-00005

18. Tos M, Lau T (1989) Hearing after surgery for cholesteatoma using various techniques. Auris Nasus Larynx 16(2):61-73. https://doi.org/10.1016/s0385-8146(89)80038-0

19. Martin AD, Harner SG (2004) Ossicular reconstruction with titanium prosthesis. Laryngoscope 114(1):61-64. https://doi.org/10. 1097/00005537-200401000-00010

20. Tomlin J, Chang D, McCutcheon B, Harris J (2013) Surgical technique and recurrence in cholesteatoma: a meta-analysis. Audiol Neurootol 18(3):135-142. https://doi.org/10.1159/000346140

21. Pontillo V, Barbara F, de Robertis V, Quaranta N (2018) Treatment of cholesteatoma with intact ossicular chain: anatomic and functional results. Acta Otorhinolaryngol Ital 38(1):61-66
22. Prasad SC, Giannuzzi A, Nahleh EA, Donato GD, Russo A, Sanna $M$ (2016) Is endoscopic ear surgery an alternative to the modified Bondy technique for limited epitympanic cholesteatoma? Eur Arch Otorhinolaryngol 273(9):2533-2540. https://doi.org/10. 1007/s00405-015-3883-3

23. Tarabichi M (2004) Endoscopic management of limited attic cholesteatoma. Laryngoscope 114(7):1157-1162. https://doi.org/10. 1097/00005537-200407000-00005

24. Smouha EE, Javidfar J (2007) Cholesteatoma in the normal hearing ear. Laryngoscope 117(5):854-858. https://doi.org/10.1097/ MLG.0b013e318033c2d6

25 Yu H, He Y, Ni Y, Wang Y, Lu N, Li H (2013) PORP vs. TORP: a meta-analysis. Eur Arch Otorhinolaryngol 270(12):3005-3017. https://doi.org/10.1007/s00405-013-2388-1

Publisher's Note Springer Nature remains neutral with regard to jurisdictional claims in published maps and institutional affiliations. 